\title{
Méthodes innovantes pour la mesure des débits fluviaux en continu : profileur Doppler fixe horizontal (H-aDcp) et analyse d'images (LSPIV)
}

\author{
Innovating methods for continuous river discharge monitoring : horizontal fixed \\ Doppler profiler (H-aDcp) and image analysis (LSPIV)
}

\author{
ALEXANDRE HAUET, JEROME LE COZ, GUILLAUME DRAMAIS \\ Cemagref, Unité de Recherche Hydrologie - Hydraulique, 3bis quai Chauveau - CP 220, 69336 Lyon cedex 9, France \\ tel. +33(0)4 722087 86, e-mail : jerome.lecoz@cemagref.fr
}

CECILE CARRE, DANIEL LEGRAS

EDF-Division Technique Générale, 21 avenue Europe, 38000 Grenoble, France

\section{GILLES PIERREFEU}

Compagnie Nationale du Rhône, Laboratoire Hydraulique et Mesures, 4 rue de Châlon-sur-Saône, 69007 Lyon, France

\section{CLAIRE GODAYER}

DIREN Rhône-Alpes, Unité Hydrométrie, Hydrologie et Hydrogéologie, 208bis rue Garibaldi 69422 Lyon cedex 3, France

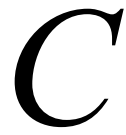

et article présente deux techniques innovantes permettant de mesurer en continu mais partiellement la vitesse d'un écoulement fluvial, et d'en déduire le débit : les profileurs Doppler fixes horizontaux (H-aDcp) mesurant les vitesses à cote fixe en travers de la section; et l'analyse d'images LSPIV mesurant les vitesses en surface de l'écoulement. Le potentiel et les limites de ces deux techniques sont illustrés par deux cas d'études : le H-aDcp installé à St-Georges sur la Saône à Lyon, et un suivi de crue par LSPIV sur l'Ardèche à Sauze - St-Martin.

$T$

his article presents two innovative techniques for discharge monitoring based on continuous though partial velocity measurements of river flows : fixed horizontal Doppler profilers (H-aDcp) measuring velocities at a constant elevation across the section; and LSPIV image analysis measuring velocities at the flow surface. The potential and limitations of both techniques are assessed through two study cases : the St-Georges H-aDcp (Saône river at Lyon) and the LSPIV monitoring of a flood in the Ardèche river at Sauze -St-Martin.

\section{INTRODUCTION}

Quantifier en continu et en temps réel le débit des rivières est une tâche délicate en termes de mise en œuvre de la mesure et d'expertise hydraulique et métrologique. Classiquement, on estime le débit d'une rivière en convertissant une mesure de hauteur d'eau en un débit dit « contrôlé » via une courbe de tarage, i.e. une relation hauteur-débit connue et univoque pour une section donnée d'un cours d'eau [1]. La représentativité de cette relation hydraulique peut parfois être mise en défaut, par exemple à cause de modifications de la géométrie du tronçon (" détarage »), ou à cause d'une perturbation du contrôle hydraulique.

En outre, les courbes de tarage doivent souvent être extrapolées aux plus forts débits, avec des incertitudes accrues par rapport à la gamme jaugée. En effet, les jaugeages embarqués (moulinet sur saumon, aDcp), depuis la berge (traille) ou un pont (camion jaugeur, cyclopotence), par dilu- tion chimique, ou encore les jaugeages de surface (saumon, flotteurs chronométrés) sont réalisables jusqu'à une certaine gamme de vitesse, de densité de flottants et de rapidité de crue, au-delà de laquelle le déploiement est compromis pour des raisons logistiques, techniques et de sécurité du matériel et surtout des opérateurs.

La mesure d'une vitesse représentative en continu, en plus de la hauteur d'eau, est très utile pour améliorer l'estimation du débit, notamment en régime influencé, fortement transitoire ou au-delà de la gamme jaugée d'une courbe de tarage. Par exemple, les systèmes ultrasoniques à temps de transit [2] fournissent la vitesse moyenne le long d'une ou de plusieurs cordes en travers de la section ; pour de petites sections, les débitmètres Doppler continus [3] fournissent la vitesse moyenne dans le faisceau acoustique de l'appareil.

Cet article présente des résultats métrologiques obtenus avec deux techniques innovantes permettant une mesure en continu mais partielle du champ de vitesse, et donc le calcul 
du débit à partir d'un profil bathymétrique et d'hypothèses hydrauliques : les profileurs Doppler fixes horizontaux $(\mathrm{H}-\mathrm{aDcp})$ mesurant les vitesses à cote fixe en travers de la section ; et l'analyse d'images LSPIV mesurant, comme la méthode par diffraction radar [4], les vitesses en surface de l'écoulement.

\section{II $\square$ PRINCIPE ET MISE EN EUVRE DE LA MESURE DES VITESSES}

\section{II.1 LE H-ADCP : PRINCIPE ET APPLICATION À ST-GEORGES (SAÔNE À LYON)}

Depuis 2005, les premiers aDcp fixes horizontaux (H-aDcp) en France $[5,6]$ ont été installés pour suivre en continu (typiquement à la minute) et en temps réel le débit au niveau de stations hydrométriques pilotes (Rhône à LyonPerrache, Saône à Lyon-St-Georges, Isère à Romans, canal d'amenée à Montélimar pour la CNR ; Rhône à St-Alban et canal d'amenée de Bollène à Tricastin pour EDF). En février 2006, la CNR a équipé la station St-Georges avec un H-aDcp Teledyne RDI $300 \mathrm{kHz}$ à 3 faisceaux «narrowbeam », i.e. avec une ouverture du lobe acoustique principal réduite $\left(0,7^{\circ}\right.$ au lieu de $3^{\circ}$ ), de manière à limiter les réflexions parasites sur la surface et surtout le fond. Le capteur est fixé en rive droite sur le mur de quai, à la cote 160,00 m NGF, 2 m environ sous le niveau de retenue habituellement observé, les faisceaux amont et aval formant un angle de $20^{\circ}$ avec le faisceau médian (Fig. 1-a).

Les composantes horizontales des vitesses en travers de la section sont calculées à partir des vitesses radiales mesurées par effet Doppler le long des trois faisceaux, pointant à $-0,58^{\circ}$ sous $1^{\prime}$ horizontale. 180 mesures acoustiques (pings) acquises sur $45 \mathrm{~s}$ sont moyennées chaque minute et enregistrées par le système, piloté par un PC industriel basse consommation alimenté par un atelier d'énergie $24 \mathrm{~V}$. Le $\mathrm{H}-\mathrm{aDcp}$ enregistre également la température, et le niveau d'eau est mesuré par une sonde de pression externe. Le logiciel VISEA-H [7] calcule le débit en temps réel à partir des vitesses mesurées et d'une bathymétrie pré-enregistrée. La télétransmission des données de débit est assurée par GPRS à la fréquence $1 \mathrm{~min}$; les fichiers de données brutes sont récupérés lors de tournées régulières.

Afin d'évaluer les performances du système en vitesse et débit, 18 jaugeages par aDcp ont été réalisés au droit de l'appareil lors de crues en février-avril 2006, pour une gamme de débit étendue (de 100 à $1800 \mathrm{~m}^{3} / \mathrm{s}$ ). Des techniques de post-traitement des données aDcp (bathymétrie et vitesses) pour établir le champ de vitesse moyen pour chaque campagne de jaugeage ont été définies, mises en œuvre et validées $[8,9]$. En particulier, il a été vérifié que les profils de vitesse moyens obtenus sont stables (variations inférieures à $5 \%$ ) pour une densité suffisante de données voisines moyennées, et que les débits calculés à partir des données post-traitées sont égaux (typiquement à $1 \%$ près) aux débits jaugés correspondants (écart moyen de $-0,3 \%$ ).

La comparaison des vitesses $\mathrm{H}-\mathrm{aDcp}$ avec les vitesses aDcp post-traitées dans le volume de mesure correspondant (Fig. 1-b) montre que [10] :

i) en moyenne les mesures $\mathrm{H}-\mathrm{aDcp}$ sont fiables (à $5 \%$ près, traits pointillés) dans un champ proche jusqu'à environ $60 \mathrm{~m}$ du capteur (sur $95 \mathrm{~m}$ de largeur de section) ; les écarts observés sont acceptables et peuvent s'expliquer par la différence dans l'échantillonnage spatial des deux techniques ;

ii) dans un champ lointain au-delà de $60 \mathrm{~m}$ du capteur, les vitesses $\mathrm{H}-\mathrm{aDcp}$ sont très rapidement fortement sousestimées ;

iii) les campagnes correspondant à des débits inférieurs à $300 \mathrm{~m}^{3} / \mathrm{s}$, soit des vitesses moyennes inférieures à $0,4 \mathrm{~m} / \mathrm{s}$ (lignes rouges épaisses) présentent une sous-estimation importante (jusqu'à - $50 \%$ pour $\mathrm{Q}=115 \mathrm{~m}^{3} / \mathrm{s}$ ) sur l'ensemble de la largeur de section.

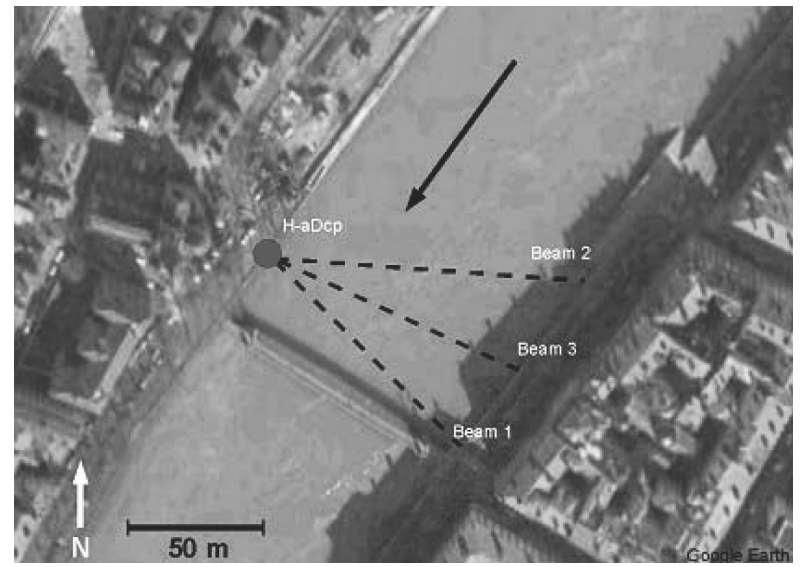

(a)

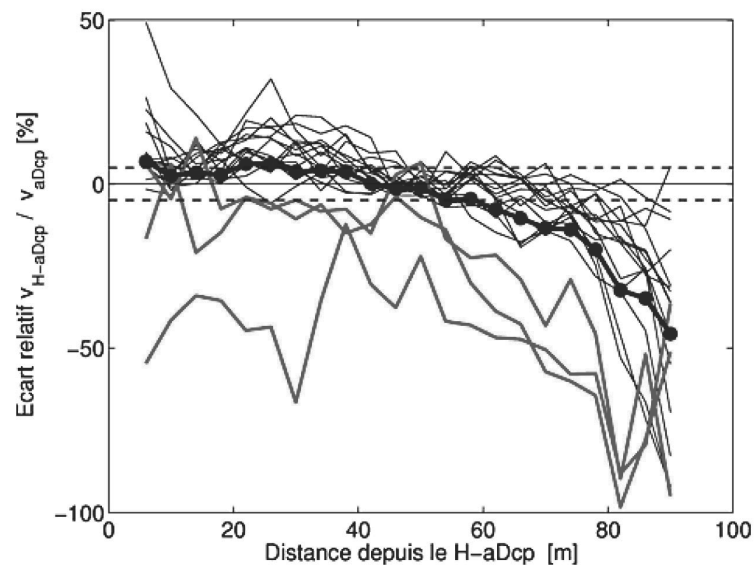

(b)

Fig. 1 - (a) Vue aérienne (Google Earth) de la station St-Georges (Saône à Lyon) avec l'emprise des trois faisceaux du H-aDcp ; (b) Ecarts relatifs des vitesses H-aDcp aux vitesses aDcp correspondantes, pour chacun des 18 jaugeages (lignes) et moyenne (points bleus). 
Des résultats similaires ont été observés fin 2007 avec un $\mathrm{H}-\mathrm{aDcp}$ identique sur l'Isère à Romans, et des travaux sont en cours pour déterminer l'origine de ces problèmes inattendus. En effet la géométrie du lobe principal des faisceaux acoustiques et celle des sections de mesure suggèrent que l'explication est plutôt à rechercher du côté des réflexions parasites des lobes secondaires sur le fond, dont la contribution à l'écho total est accrue en présence de faibles vitesses et surtout de faibles concentrations en matières en suspension. Ainsi la technologie $\mathrm{H}-\mathrm{aDcp}$ semble fiable et prometteuse pour l'estimation des débits moyens à forts, mais à bas débit (lorsque la concentration est faible), des développements nécessaires sont en cours. Lors de la forte crue de l'Isère en juin 2008, il a cependant été observé que de fortes concentrations de sédiments en suspension pouvaient réduire drastiquement la portée utile par atténuation du signal acoustique.

\section{II.2 LA LSPIV : PRINCIPE ET APPLICATION À SAUZE-ST-MARTIN (ARDÈCHE)}

L'analyse d'images permet de mesurer le champ 2D de vitesse en surface d'un écoulement à condition que des traceurs visibles, tels que des particules solides (débris végétaux, petits flottants, etc.), des bulles ou des figures de turbulence soient advectés avec l'écoulement. Cette technique est dérivée de la Particle Image Velocimetry (PIV, voir [11]) utilisée en laboratoire, mais pour une étude sur des objets de grandes échelles de type rivière, d'où le nom de Large-Scale PIV (LSPIV) [12]. Une mesure LSPIV comprend l'enregistrement d'une séquence d'images horodatées de l'écoulement, une correction géométrique des images pour s'affranchir des effets de distorsion de perspective (orthorectification) et un calcul du déplacement des traceurs de l'écoulement grâce à une analyse statistique en corrélation des motifs. Connaissant la bathymétrie d'une section en travers et supposant un modèle de distribution verticale de vitesses, on peut arriver à l'estimation du débit à partir du champ de vitesse LSPIV. La LSPIV a été utilisée pour estimer des débits de rivières d'échelles très différentes, des étiages [13] aux fortes crues [14] ainsi que pour l'amélioration de courbes de tarage en régimes hydrauliques normaux $[15,16]$. La LSPIV ne peut être mise en œuvre de nuit (essais par caméra infrarouge), en cas de brouillard, ou si des obstacles ou salissures (toiles d'araignée...) gênent le capteur. En contrepartie de ces limitations techniques, le matériel est peu coûteux et facile à installer.

Cet article présente les résultats préliminaires d'un projet en cours, au Cemagref de Lyon (unité de recherche Hydrologie / Hydraulique) en collaboration avec la Compagnie Nationale du Rhône (CNR) et l'Observatoire Hydrométéorologique Méditerranéen - Cévennes Vivarais (OHM-CV), visant à installer un réseau de stations débitmétriques LSPIV sur le bassin de l'Ardèche. L'Ardèche, en raison de caractéristiques synoptiques atmosphériques, de sa géologie et de son relief, est sujette aux crues-éclairs, c'est-à-dire à des pics de crue survenant très rapidement après le maximum des précipitations. De plus, l'Ardèche est une rivière engendrant des volumes de crue considérables par rapport aux débits courants : à Vogüé, alors que le module interannuel est de $26 \mathrm{~m}^{3} / \mathrm{s}$, la crue de période de retour 10 ans atteint $1400 \mathrm{~m}^{3} / \mathrm{s}$ (Fiche station V5014010 Banque Hydro), soit un rapport d'environ 50. Pour ces raisons, mesurer les crues en Ardèche est crucial mais difficile. Les courbes de tarage sont souvent extrapolées pour les plus forts débits, ce qui rend les estimations délicates. Le contrôle par modélisation hydraulique de la partie haute des courbes de tarage permet d'en vérifier la bonne cohérence lorsque des laisses de crue sont disponibles au voisinage de la station hydrométrique [17]. Un réseau de stations LSPIV doublant les stations hydrométriques déjà présentes apportera des mesures de vitesse à fort débit qui permettront de diminuer l'incertitude associée aux estimations de débit des fortes crues.

Pour vérifier les capacités de la LSPIV à mesurer pendant les crues, une expérimentation " mobile » a été menée lors de la crue de l'Ardèche les 22 et 23 novembre 2007, au niveau de la station CNR / EDF / DIREN RA / SPC-GD de Sauze-St Martin d'Ardèche. La station de Sauze a été choisie car de nombreux jaugeages sont disponibles, pour une gamme de débits étendue (jusqu'à $2700 \mathrm{~m}^{3} / \mathrm{s}$ ). La Fig. 2-a montre l'hydrogramme (à pas de temps variable) de cette crue fourni par la courbe de tarage CNR, avec un pic d'environ $900 \mathrm{~m}^{3} / \mathrm{s}$ atteint le 23 au matin. Les zones grisées sur la figure représentent les périodes de mesure LSPIV. Ces mesures ont été réalisées grâce à un mât télescopique d'une hauteur maximale de 10 mètres sur lequel une plate-forme orientable et une caméra sont fixées (Fig. 2-b, [18]). Un ordinateur au sol permet de régler l'orientation de la caméra et les paramètres de l'enregistrement des images. Le capteur utilisé est un simple camescope numérique mini-DV, de résolution $720 \times 576$ pixels, disponible dans le commerce à un faible prix (environ $300 €$ ). Les mesures du 22 novembre ont été réalisées en même temps qu'une campagne de jaugeages par aDcp de la CNR.

L'étude d'écoulements de grande échelle comme les cours d'eau nécessite l'enregistrement d'images sur de grandes surfaces, souvent supérieures à $10000 \mathrm{~m}^{2}$. Les images sont généralement prises depuis les berges de la rivière avec un angle de site très important, ce qui implique une distorsion due à l'effet de perspective. La première étape de l'analyse LSPIV consiste à corriger, ou orthorectifier, les images grâce à un jeu d'équations photogrammétriques reliant le système de coordonnées 3D $[X, Y, Z]$ de l'objet naturel d'étude au système de coordonnées $2 \mathrm{D}[i, j]$ des images par le biais de points d'amarres de coordonnées connues à la fois dans l'image et dans l'espace réel (voir [19]) :

$$
\begin{aligned}
& i=\frac{a_{1} X+a_{2} Y+a_{3} Z+a_{4}}{a_{9} X+a_{10} Y+a_{11} Z+1} \\
& j=\frac{a_{5} X+a_{6} Y+a_{7} Z+a_{8}}{a_{9} X+a_{10} Y+a_{11} Z+1}
\end{aligned}
$$

Les coefficients $a_{i}$ n'ont pas de sens physique mais sont des combinaisons des paramètres intrinsèques (distance focale, taille du capteur) et extrinsèques (position et orientation) de la caméra. 
La Fig. 3-a montre une image orthorectifiée du site de Sauze lors de la crue du 22 novembre 2007. Une fois les images orthorectifiées, une analyse statistique de reconnaissance des déplacements de traceurs en surface de l'écoulement est appliquée. Elle consiste à calculer la corrélation entre une fenêtre d'interrogation (FI) centrée sur un point $a$ dans une image 1 prise au temps $t$, et une même FI centrée sur un point $b$ dans une image 2 prise à $t+\Delta t$. Un coefficient de corrélation croisée $R(a, b)$ est utilisé comme index de similarité entre les formes incluses dans les deux FI. Le déplacement le plus probable du fluide, à partir du point $a$ et durant la période $\Delta t$ est le point $b$ pour lequel le coefficient de corrélation est maximal. Grâce à un ajustement parabo- lique, Fujita et Komura [20] ont montré que l'on pouvait mesurer des déplacements avec une résolution de 0,2 pixel. En divisant les déplacements identifiés par $\Delta t$, on estime une vitesse à chaque nœud de calcul. Il apparaît donc que la taille de la FI est un paramètre clé car elle règle la résolution de la mesure. Elle doit être suffisamment petite pour préserver l'échelle intéressante de l'écoulement (toutes les échelles inférieures seront moyennées pendant l'analyse statistique), mais suffisamment grande pour inclure des traceurs visibles.

$\mathrm{Vu}$ l'approche purement statistique utilisée pour le calcul des déplacements, il est possible d'obtenir des vectrices vitesses erronés. De nombreuses routines de post-traitement existent pour filtrer ces vecteurs erronés, à partir de seuils

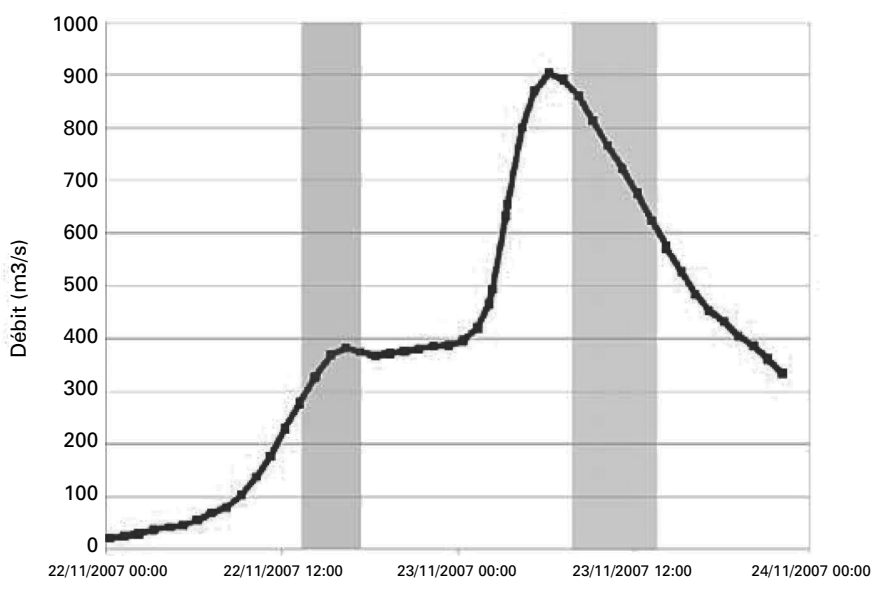

(a)

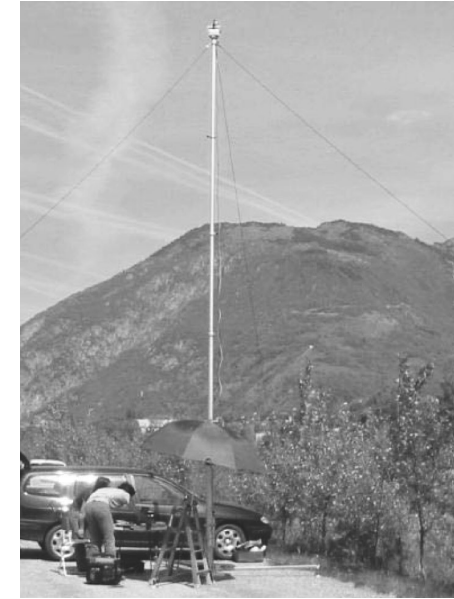

(b)

Fig. 2 - (a) Hydrogramme de la crue de l'Ardèche des 22 et 23 novembre à Sauze - St Martin fourni par la courbe de tarage CNR et (b) dispositif mobile pour les mesures LSPIV lors d'un déploiement sur l'Arc en Maurienne [17].

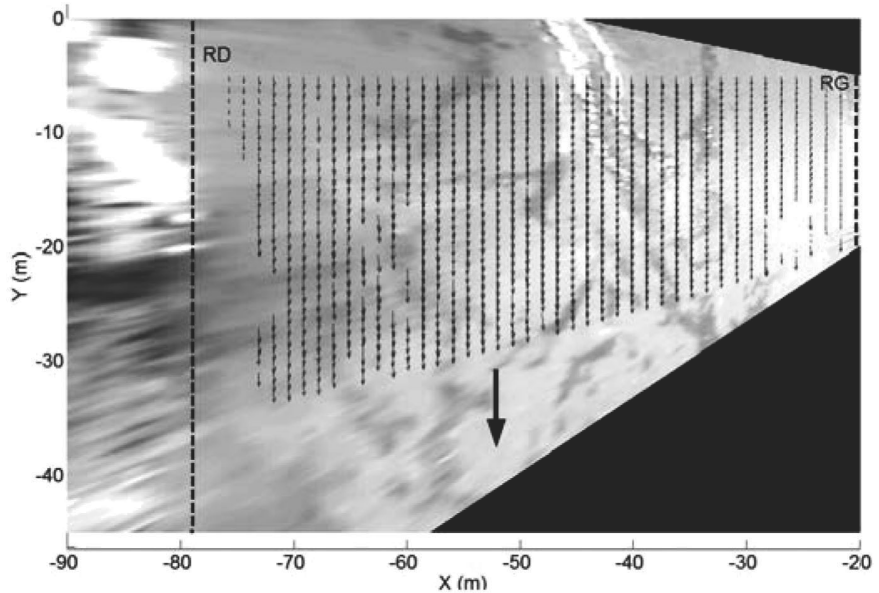

(a)

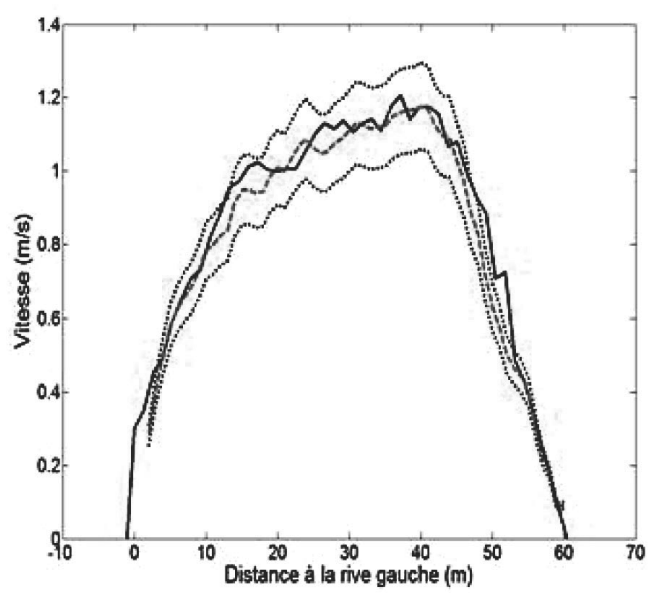

(b)

Fig. 3 - (a) Image orthorectifiée de l'écoulement à Sauze et champ de vitesse en surface calculé par LSPIV. Les traits pointillés localisent les berges droite et gauche ; (b) Vitesses moyennées sur la profondeur mesurées par aDcp (traits pointillés avec écarts à $10 \%$ ) et LSPIV (trait plein) le long du transect de mesure aDcp. 
sur le coefficient de corrélation, sur l'intensité ou la direction des vitesses, ou encore d'une analyse de continuité de l'écoulement, par exemple. La Fig. 3-a présente un champ de vitesse de surface calculé par PIV à Sauze. Les vitesses ont été moyennées sur une séquence de 2 minutes avec 5 images par seconde (600 images).

La Fig. 3-b présente la comparaison des vitesses mesurées (i) par LSPIV et (ii) par aDcp (mesures réalisées par la CNR, équipe de Th. Pantel) le long du transect de mesure aDcp. A partir de 12 sections en travers de mesure aDcp, un champ de vitesse moyen a été établi (pour la méthode, voir $[8,9])$, et un profil transversal de vitesses moyennes sur la hauteur d'eau a été calculé. Pour la même section en travers (correspondant à $\mathrm{Y}=-15 \mathrm{~m}$ sur la Fig. 3-a), les vitesses LSPIV sont extraites. Pour pouvoir comparer aDcp et LSPIV, un coefficient correcteur de 0,90 , correspondant au rapport entre la vitesse moyenne sur la hauteur d'eau et la vitesse de surface, est affecté aux vitesses LSPIV. La valeur de ce coefficient sera discutée en partie III.

On observe une très bonne adéquation entre les profils de vitesse mesurés par aDcp et LSPIV et ce sur toute la gamme de vitesse (de 0,1 à $1,2 \mathrm{~m} / \mathrm{s}$ ). La vitesse LSPIV ne s'écarte quasiment jamais à plus de $10 \%$ de la mesure aDcp prise comme référence. On note cependant une sur-estimation des vitesses LSPIV plus importante en rive droite, c'est-à-dire dans le champ lointain de la caméra, où la diminution de la résolution de la caméra entraîne des erreurs.

\section{M MÉTHODES DE CALCUL DU DÉBIT}

\section{III.1 H-ADCP : MÉTHODE DE LA VITESSE INDEX ET MÉTHODE DU PROFIL DE VITESSE}

Différentes méthodes de calcul du débit total à partir des vitesses mesurées par $\mathrm{H}-\mathrm{aDcp}$ sont envisageables. La Méthode de la Vitesse Index (MVI) est la plus simple à mettre en œuvre : une corrélation empirique est recherchée par régression linéaire de la vitesse débitante obtenue par jaugeage contre une vitesse index établie à partir des vitesses H-aDcp [21]. Le plus simple est de définir la vitesse index comme la moyenne des vitesses $\mathrm{H}-\mathrm{aDcp}$ mesurées sur une portion du profil horizontal. L'inconvénient de cette méthode est la nécessité de disposer d'un échantillon suffisant de jaugeages pour caler la corrélation sur toute la gamme utile.

L'autre méthode actuellement proposée par différents logiciels commerciaux est la Méthode du Profil de Vitesse (MPV) : pour chaque cellule de mesure du H-aDcp, la vitesse moyenne sur la verticale est calculée à partir d'une loi de distribution verticale des vitesses. Cette méthode est plus délicate à mettre en œuvre que la MVI, mais ses fondements physiques permettent un calage a priori (puis vérifications par jaugeages), et son extrapolation en dehors de la gamme jaugée est potentiellement plus robuste. Les profils théoriques envisageables sont les lois puissance, loi de paroi logarithmique [21], et des variantes de la loi log, comme le profil « log-constant» (profil log puis constant au-dessus d'une cote relative donnée) et le profil de Van Rijn [7,23] :

$$
\begin{aligned}
\frac{v_{z}}{v_{\text {surf }}} & =A_{1} \cdot \ln \left(\frac{z}{z_{0}}\right)+ \\
& {\left[1-A_{1} \ln \left(\frac{h}{z_{0}}\right)\right] \cdot\left[2\left(\frac{z-z_{0}}{h-z_{0}}\right)^{t}-\left(\frac{z-z_{0}}{h-z_{0}}\right)^{2 t}\right] }
\end{aligned}
$$

avec $v_{z}$ la vitesse à la cote $z$ au-dessus du fond, $v_{\text {surf }}$ la vitesse en surface, $h$ le tirant d'eau, $z_{0}$ la cote où la vitesse s'annule ( hauteur de rugosité » fonction du substrat), $A_{1}$ un paramètre permettant de doser la perturbation apportée au profil log, et $t$ un paramètre calculé de sorte que $v_{z=h / 2}$ soit indépendant de la valeur de $A_{1}$. Par la suite, $V$ désigne la vitesse moyennée sur la hauteur d'eau.

Comme on l'a vu, il est rare que le H-aDcp puisse scruter convenablement les vitesses sur toute la largeur de section. Avec la MPV, il est donc nécessaire de choisir une stratégie d'extrapolation des vitesses moyennes dans le champ lointain inexploré ou inexploitable. Parmi les stratégies envisageables, l'extrapolation peut se fonder sur un profil transversal moyen des vitesses en travers de la section, sur une hypothèse de Froude constant en travers de la section [24], ou plus simplement sur une hypothèse de vitesse débitante équivalente sur la sous-section mesurée et la sous-section extrapolée.

Le paramétrage des différentes techniques de calcul de débit gagne à être appuyé sur une analyse de la structure du champ de vitesse, comme celle réalisée à St-Georges à partir des jaugeages aDcp post-traités. En particulier, la zone d'utilisation des vitesses $\mathrm{H}-\mathrm{aDcp}$ pour la MVI comme pour la MPV a été limitée aux cellules situées de $6 \mathrm{~m}$ à $58 \mathrm{~m}$ $\mathrm{du}$ capteur, sur une largeur totale de $95 \mathrm{~m}$. L'analyse des profils verticaux de vitesse (Fig. 4-a-b) a permis d'établir le paramétrage des quatre lois de profil vertical citées [10]. En particulier la loi $\log \left(z_{0}=0,0046 \mathrm{~m}\right)$ représente bien le profil moyen en-dessous de la cote relative $z / h=0,7$, le profil étant plutôt constant au-dessus. La loi de Van Rijn ( $z_{0}=0,0046 \mathrm{~m}, A_{1}=0,075$ ) permet de représenter le profil moyen sur toute la hauteur d'eau de façon acceptable.

\section{III.2 LSPIV : MÉTHODE DU COEFFICIENT DE SURFACE}

La LSPIV ne permet d'accéder qu'au champ de vitesse à la surface libre. Pour calculer un débit, on doit utiliser un modèle de distribution verticale des vitesses pour calculer une vitesse moyenne sur la hauteur d'eau à partir de la vitesse de surface. Couramment, on suppose que la distribution verticale des vitesses suit une loi logarithmique ou une loi puissance en $1 / \mathrm{m}$ (avec $\mathrm{m}=6$ ou 7 pour les conditions typiques de rugosité), d'où un rapport $\alpha$ entre la vitesse moyenne et la vitesse de surface d'environ 0,85 pour les conditions usuelles de rugosité [4]. Dans la mesure du possible, il convient de vérifier cette hypothèse par des mesures de profils verticaux de vitesse. 
Dans l'étude de la crue de l'Ardèche à Sauze le 22 novembre 2007, les profils aDcp ont été normalisés (par le tirant d'eau et la vitesse moyenne sur la hauteur d'eau). On obtient une distribution «type » sur laquelle un modèle de profil peut être calé. La forme de ce profil donne une valeur expérimentale du coefficient a. Comme illustré en Fig. 5-a, les profils ont en moyenne une forme logarithmique sur la zone profonde de la tranche d'eau $(z / h<0,6)$, puis sont quasiment constants jusqu'à la dernière mesure (autour de $z / h=0,9$ ). En prolongeant le profil jusqu'à la surface libre, on obtient $v_{\text {surf }} / V=1,11$ soit $\alpha=0,90$. Cette valeur sera donc utilisée pour calculer les vitesses moyennes à partir des vitesses LSPIV : $V=\alpha \cdot V_{L S P I V}=0,90 \cdot V_{L S P I V}$.
Cette valeur de a est comparée, sur 29 jaugeages au moulinet de la CNR, à la valeur du rapport entre le débit calculé en utilisant toutes les mesures de vitesse (jaugeages complets), $Q$, et le débit calculé en utilisant seulement les vitesses à $20 \mathrm{~cm}$ sous la surface libre, Q2. Cette vitesse à $20 \mathrm{~cm}$ est assimilée à la vitesse en surface dans les protocoles de mesure de la CNR, pour des questions de mise en œuvre. Comme illustré en Fig. 5-b, on observe que le rapport $Q / Q 2$ évolue entre 0,79 et 0,99 , avec une moyenne de 0,89 et un écart-type expérimental de 0,05 . La valeur moyenne de $Q /$ Q2 est cohérente avec celle obtenue pour $\alpha$, même si on constate une dispersion assez forte, surtout pour les petits débits.

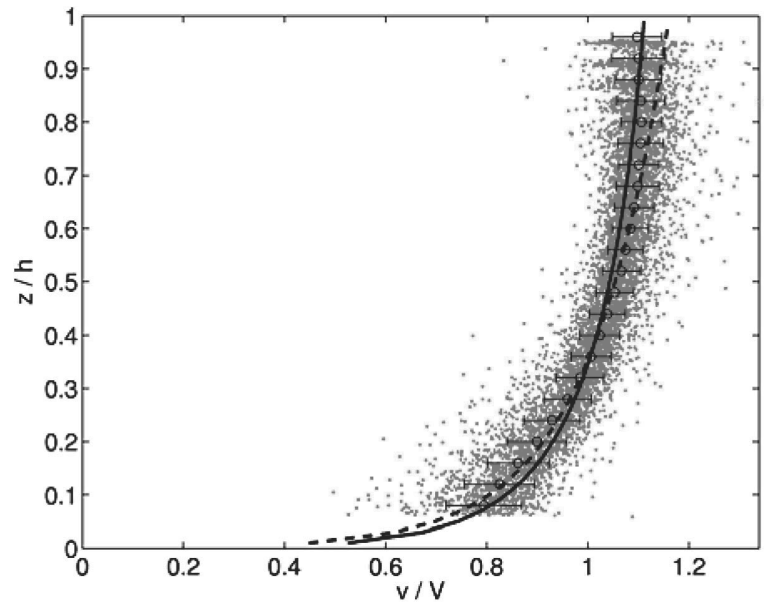

(a)

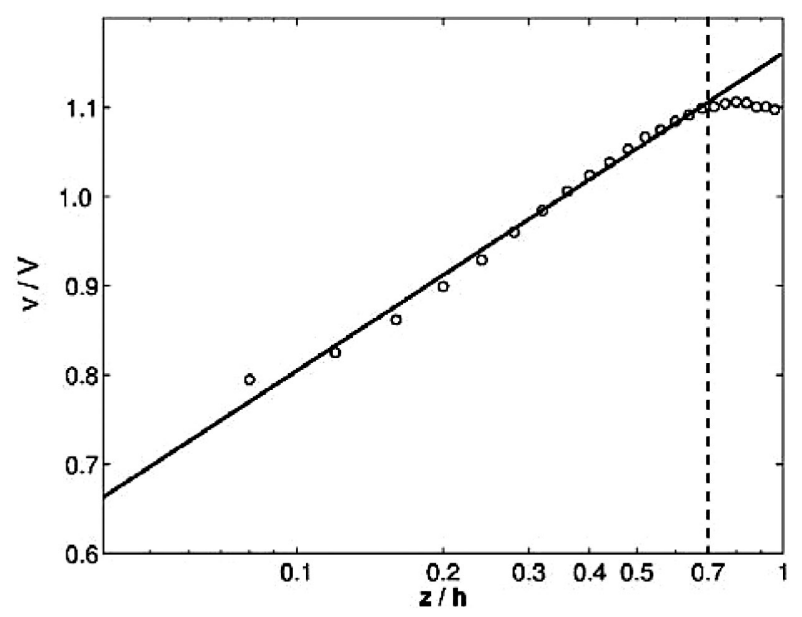

(b)

Fig. 4 - a) Profils adimensionnels de vitesse aDcp post-traités : nuage de points des 414 profils, profil moyen et écarttype expérimental (cercles), profil log calé (pointillés), profil de Van Rijn calé (ligne épaisse) ;

b) Profil adimensionnel moyen (cercles) et calage logarithmique valable jusqu'à $\mathbf{z} / \mathbf{h}=\mathbf{0 , 7}$.
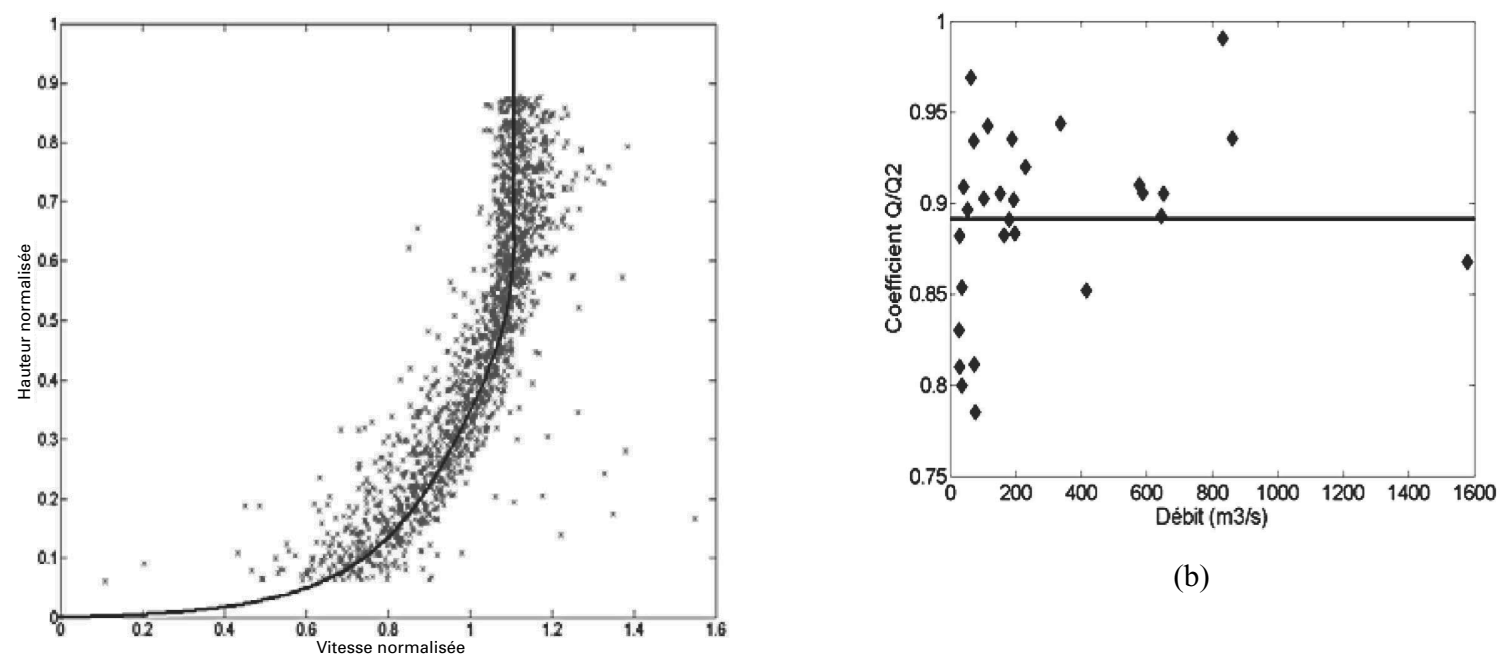

(b)

(a)

Figure 5 - (a) Profils adimensionnels de vitesse aDcp post-traités (points bleus) et profil log-constant calé (trait noir) ; (b) Rapport Q/Q2 pour 29 jaugeages CNR au moulinet à Sauze. 


\section{RÉSULTATS : CONFRONTATION À DES MESURES DE DÉBIT INDÉPENDANTES}

\section{IV.1 DÉBITS H-ADCP À ST GEORGES}

Les différentes méthodes MVI (Méthode de la Vitesse Index), MPV (Méthode des Profils Verticaux) champ proche, et MPV totale avec extrapolation en champ lointain ont été appliquées aux vitesses mesurées par le H-aDcp pendant les jaugeages aDcp [10]. Ces calculs de test ont été réalisés avec Matlab, certaines options pouvant être implémentées sur les logiciels commerciaux. Les résidus (écarts relatifs des valeurs calculées aux valeurs de référence issues des 18 jaugeages) sont présentés Fig. 6 .

La MVI (Fig. 6-a) fournit des débits acceptables (écarts $<5 \%$ typiquement) pour toute la gamme de débit explorée, sauf pour la campagne à $115 \mathrm{~m}^{3} / \mathrm{s}(-34 \%)$ à cause de la sous-estimation forte des vitesses H-aDcp sur toute la section à bas débit. La régression linéaire compense dans une certaine mesure la sous-estimation des vitesses pour $Q<300 \mathrm{~m}^{3} / \mathrm{s}$ à Saint-Georges. Cette compensation n'est toutefois pas physique, et des vérifications avec de nombreux jaugeages dans les conditions de bas débit seraient nécessaires pour en assurer la validité.

Il convient d'évaluer les résultats de la MPV d'abord sans extrapolation, dans la partie de la section scrutée correctement par le H-aDcp $(0-60 \mathrm{~m})$. Pour $Q>300 \mathrm{~m}^{3} / \mathrm{s}$, la MPV (Fig. 6-b) fournit des vitesses débitantes sur la sous-section de champ proche (sans extrapolation) qui sont en accord acceptable (écarts $<5 \%$ typiquement) avec les vitesses débitantes aDcp correspondantes. On remarque cependant une tendance moyenne à la surestimation (quelques \%). Logiquement, la sous-estimation des vitesses H-aDcp pour les 3 campagnes à $Q<300 \mathrm{~m}^{3} / \mathrm{s}$ n'est pas compensée. Le choix du profil théorique (ici log-constant) influe assez peu sur les résultats.
Le choix de la méthode d'extrapolation en champ lointain utilisée pour calculer le débit total peut entraîner des variations significatives des résidus. En général, les résultats restent bons pour $\mathrm{Q}>300 \mathrm{~m}^{3} / \mathrm{s}$, comme le montre la Fig. 6-c pour un choix d'extrapolation des vitesses à Froude constant. La sous-estimation pour $Q<300 \mathrm{~m}^{3} / \mathrm{s}$ n'est naturellement pas compensée par l'extrapolation en champ lointain. Rappelons que la limite de débit $\left(300 \mathrm{~m}^{3} / \mathrm{s}\right)$ ou vitesse $(0,4 \mathrm{~m} / \mathrm{s})$ en-deçà de laquelle la mesure est faussée est spécifique du site considéré, et que le facteur limitant est vraisemblablement lié à la concentration en particules en suspension.

\section{IV.2 DÉBITS LSPIV À SAUZE}

A Sauze, le lit de la rivière est très stable puisqu'il est formé de grandes plaques calcaires. Il y a donc très peu d'érosion et il n'y a pas de dépôts sédimentaires visibles, susceptibles de changer la géométrie en cours de crue. La bathymétrie mesurée par la CNR à l'aDcp lors de la campagne du 22 novembre est utilisée par la suite. Le long d'une section en travers, ici la section mesurée à l'aDcp par la CNR, une vitesse de surface LSPIV Vsurf(i) est associée à chaque point $i$ de bathymétrie connue $d(i)$. Cette vitesse est la moyenne, pondérée par l'inverse de la distance, des 4 vitesses LSPIV les plus proches du point $i$. A partir de la vitesse de surface $\operatorname{Vsurf}(i)$ est calculée une vitesse moyenne sur la hauteur d'eau Vmoy $(i)$ grâce au coefficient de surface $\alpha$. En associant la distance entre $i+1$ et $i-1, \operatorname{Vmoy}(i)$ et $d(i)$ on calcule un débit partiel pour le point $i$. Le débit total est la somme des débits partiels sur la section en travers.

Le 22 novembre 2007, deux jaugeages aDcp et LSPIV ont été réalisés en simultané. Le Tab. 1 présente la comparaison de ces jaugeages aux estimations de débit via la courbe de tarage de la CNR. Les débits aDcp et LSPIV sont proches de la courbe de tarage (différence maximale de 3,4 \%) et sont très cohérents entre eux (différence maximale de 2,6\%). Il faut garder à l'esprit que l'incertitude sur un jaugeage aDcp

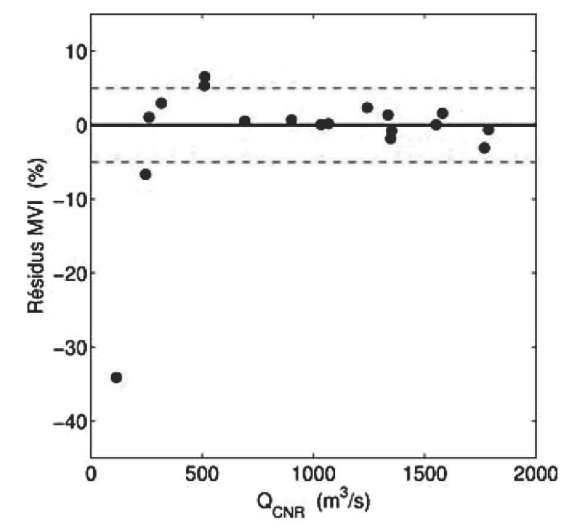

(a)

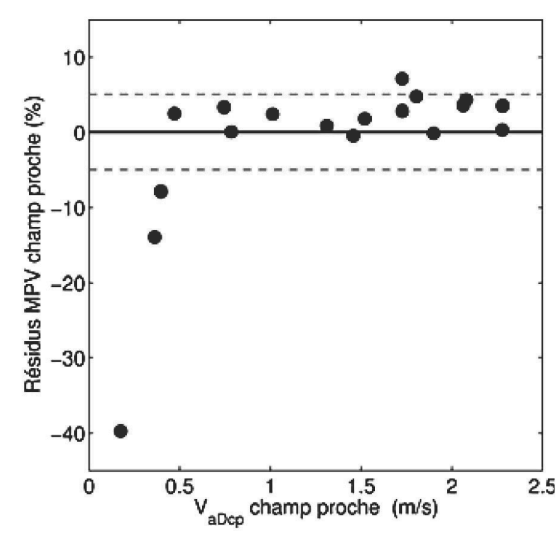

(b)

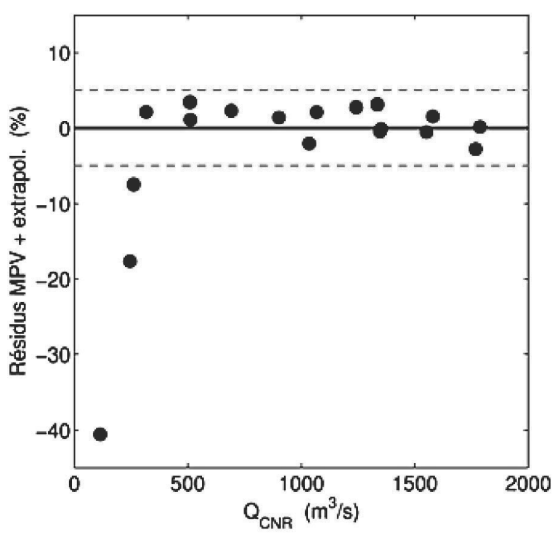

(c)

Fig. 6 - Résidus en débits/vitesses obtenus pour les différentes méthodes, par rapport aux valeurs jaugées : a) débits MVI ; b) vitesses moyennes MPV sur la sous-section scrutée correctement (profil log-constant) ; c) débits MPV (profil log-constant, avec extrapolation des vitesses à Froude constant en champ lointain). 
est typiquement de $5 \%$, et que l'incertitude LSPIV n'est certainement pas moindre que pour un jaugeage complet.

Le 23 novembre, une nouvelle mesure a été réalisée par LSPIV (mais sans jaugeage aDcp). Avec des vitesses de surface maximales atteignant plus de $3 \mathrm{~m} / \mathrm{s}$, le débit calculé en utilisant une valeur de $\alpha$ toujours prise égale à 0,90 est de $825 \mathrm{~m}^{3} / \mathrm{s}$. Ce débit surestime de $8 \%$ le débit de référence, interpolé à partir de l'hydrogramme instantané à pas de temps variable de la CNR $\left(760 \mathrm{~m}^{3} / \mathrm{s}\right)$. Bien que cette différence de $8 \%$ soit acceptable, il est intéressant de comprendre quelles sont les sources d'erreur possibles. Tout d'abord, il se peut que le coefficient $\alpha$ ait varié avec le changement de conditions hydrauliques. Ensuite, il faut considérer l'incertitude liée à la LSPIV. Nous avons choisi d'estimer le débit à partir d'une séquence vidéo de 5 minutes, qui correspond à un temps suffisamment long pour moyenner les erreurs aléatoires affectant la mesure LSPIV, et suffisamment court pour ne pas recouvrir une gamme de débit importante (le gradient de décrue étant important). Enfin, $800 \mathrm{~m}^{3} / \mathrm{s}$ représentent un débit assez élevé, pour lequel les jaugeages sont plus rares. L'incertitude moyenne sur une courbe de tarage, estimée par Olivier et al. [25] à $9 \%$ au minimum dans la gamme jaugée, est donc également à prendre en compte dans l'écart avec l'estimation LSPIV. Des essais complémentaires permettront d'avancer sur cette question.

\section{CONCLUSIONS ET PERSPECTIVES}

Deux techniques innovantes permettent de mesurer en continu mais partiellement la vitesse d'un écoulement fluvial, et d'en déduire le débit : les profileurs Doppler fixes horizontaux $(\mathrm{H}-\mathrm{aDcp})$ mesurant les vitesses à cote fixe en travers de la section; et l'analyse d'images LSPIV mesurant les vitesses en surface de l'écoulement. Le potentiel et les limites de ces deux techniques ont été illustrés par deux cas d'études : le H-aDcp installé à St-Georges sur la Saône à Lyon, et un suivi de crue par LSPIV sur l'Ardèche à Sauze - St-Martin. Les exemples proposés vérifient la fiabilité des mesures H-aDcp et LSPIV pour des conditions de débit normales à assez fortes en des sites où la courbe de tarage est bien connue. A basses eaux, les deux méthodes souffrent du manque de traceurs de l'écoulement. Néanmoins, ces techniques ont un grand intérêt pour les très forts débits peu ou pas jaugés, pour lesquels l'incertitude sur le coefficient de vitesse (ou sur la loi de profil de vitesse) deviendra négligeable face à l'incertitude de l'extrapolation des courbes de tarage [17].
L'estimation de débit par H-aDcp et LSPIV se heurte au même problème de la connaissance partielle du champ de vitesse, qui peut être résolu en considérant un profil théorique de vitesse pour calculer le débit. Sur les deux exemples présentés, le profil vertical adimensionnel type est établi à partir de jaugeages aDcp post-traités et prend une forme logarithmique puis quasi-constante pour $z / h>0,6-0,7$. Le travail d'analyse des profils verticaux de vitesse sera poursuivi pour différents sites et différents débits. Pour la LSPIV, une station en développement sur le site DIREN RA de Chazey-sur-Ain permettra de comparer le coefficient de surface à celui trouvé en Ardèche. Plus globalement, il serait intéressant de relier la valeur de ce coefficient ou encore la forme moyenne des profils verticaux à des paramètres géométriques et hydrauliques des cours d'eau, pour un paramétrage a priori du calcul de débit H-aDcp ou LSPIV.

Le couplage des mesures de vitesse par LSPIV ou H-aDcp à une modélisation numérique $2 \mathrm{D}$ du tronçon constitue une piste pour améliorer l'estimation de débit, quand la mesure ne peut être effectuée sur la totalité de la section (portée limitée du H-aDcp et de la LSPIV, manque de traceurs pour la LSPIV). L'extrapolation du champ de vitesse mesuré à l'ensemble de la section peut s'appuyer sur le modèle, une fois calé. Pour la LSPIV, cette étude est en cours de réalisation, avec le modèle Rubar20 (Cemagref), pour les stations de Chazey-sur-Ain et de Sauze.

Le H-aDcp et la LSPIV étant des techniques émergentes, il apparaît nécessaire de poursuivre l'effort de caractérisation des sources d'incertitudes et de quantification de leur impact sur les estimations de débit. Hauet et al. [26] ont développé en ce sens un simulateur numérique de la LSPIV permettant des tests de sensibilité sur les différents composants de la mesure par imagerie : enregistrement des images, orthorectification, mesure de la hauteur d'eau, de la bathymétrie, coefficient de surface, etc. Un tel simulateur pourrait aussi être développé pour le cas des H-aDcp, une fois expliquée et corrigée la sous-estimation des vitesses observée en champ lointain et à bas débit.

\section{VI — REMERCIEMENTS}

L'étude présentée ici est fondée sur le travail et la collaboration de nombreux techniciens et ingénieurs des équipes hydrométriques de la CNR, du Cemagref, d'EDF-DTG et des services de l'Etat. Qu'ils en soient chaleureusement remerciés ici.

Tableau 1 - Comparaison des débits aDcp / LSPIV à ceux donnés par la courbe de tarage CNR.

\begin{tabular}{|l|c|c|c|c|}
\hline \multicolumn{1}{|c|}{ Méthode } & Jaugeage 1 $\left(\mathbf{m}^{\mathbf{3}} / \mathbf{s}\right)$ & Différence $\mathbf{( \% )}$ & Jaugeage 2 $\mathbf{( \mathbf { m } ^ { 3 } / \mathbf { s } )}$ & Différence $\mathbf{( \% )}$ \\
\hline Courbe de tarage & 320 & - & 343 & - \\
\hline aDcp - CNR & 327 & $+2,1$ & 345 & $+0,6$ \\
\hline LSPIV - Cemagref & 331 & $+3,4$ & 336 & $-2,1$ \\
\hline
\end{tabular}




\section{Q RÉFÉRENCES}

[1] Perret C. (2008) - Les moyens de contrôle et de mesure des débits. Les capteurs et les méthodes. Colloque SHF « Mesures hydrologiques et incertitudes », 1-2 avril 2008, Paris, France.

[2] Lengricht J., Stephan G., Frey W. (2007) - Venice lagoon monitoring with wireless acoustic transit time flowmeters synced by GPS and Bluetooth. 32e Congrès de l'AIRH, Venise, Italie.

[3] Birgand F., Benoist J.-C., Novince E., Gilliet N., SaintCAst P., Le SAOs E. (2005) - Mesure des débits à l'aide de débitmètres ultrasoniques Doppler - Cas des petits cours d'eau ruraux. Ingénieries. EAT. 41 23-38

[4] Costa J. E., Cheng R. T., Haeni F. P., Melcher N., Spicer K. R., Hayes E., Plant W., Hayes K., Teague C., Barrick D. (2006) - Use of radars to monitor stream discharge by noncontact methods, W07422, doi10.1029/2005WR004430. Water Resources Research. 42 (7)

[5] Pierrefeu G. (2006) - Monitoring the Saône river discharge in Lyon by H-ADCP. TRDI "ADCPs in Action", Cannes, France.

[6] Legras D. (2006) - Setting up of a $300 \mathrm{kHz}$ narrowbeam H-ADCP upstream Saint Alban nuclear power plant on the Rhone river. TRDI "ADCPs in Action", Cannes, France.

[7] (2003) - Version. Visea-H Manual 2.xx, Aqua Vision BV, PaysBas.

[8] Le Coz J., Paquier A., Chastan B., Dramais G. (2007) Emploi des profileurs acoustiques à effet Doppler ( $\mathrm{aDcp})$ pour étudier la structure des écoulements en rivière. $18^{e}$ Congrès français de Mécanique, Grenoble, France.

[9] Le Coz J., Pierrefeu G., Jodeau M., Paquier A. (2007) Mean vertical velocity profiles from aDcp river discharge measurement datasets. $32^{e}$ Congrès de l'AIRH, Venise, Italie.

[10] Le Coz J., Pierrefeu G., Paquier A. (2008) - Evaluation of river discharges monitored by a fixed side-looking Doppler profiler (H-aDcp)., W00D09, doi : 10.1029/2008WR006967. Water ressources Research. 44

[11] AdRIAN R.J. (2005) — Twenty years of particle image velocimetry. Experiments in Fluids. 39 159-169

[12] Muste, Muste M., Fujita I., Hauet A. (2008) - Largescale particle image velocimetry for measurements in riverine environments, doi10.1029/2008WR006967. Water Resources Research, W00D19. 44

[13] Bradley A. A., Kruger A., Mesehle E. A., Muste M. V. I (2002) - Flow Measurement in Streams Using Video Imagery, doi 10.1029/2002WR001317. Water Resources Research. 38(12)
[14] Fujta I., Muste M., Kruger A. (1998) - Large-scale particle image velocimetry for flow analysis in hydraulic engineering applications. Journal of Hydraulic Research. 36(3) 397 414

[15] Creutin J.D., Muste M., Bradley A. A., Kim S. C., Kruger A. (2003) - River Gauging Using PIV Techniques : A Proof of Concept Experiment on the Iowa River. Journal of Hydrology. 277(3-4) 182-194

[16] Hauet A., Kruger A., Krajewski W.F., Bradley A.A., Muste M., Creutin J.-D., Wilson M. (2008) - An experimental system for real-time discharge estimation using an image-based method. ASCE Journal of Hydrologic Engineering. 13(2) 105110

[17] Pobanz K., Renouf E., Sauquet E., Lang M. (2008) Extrapolation des courbes de tarage en crue par modélisation hydraulique. Colloque SHF « Mesures hydrologiques et incertitudes ", 1-2 avril 2008, Paris, France.

[18] Jodeau M., Hauet A., Paquier A., Le Coz J., Dramais G. (2008) - Application and evaluation of LS-PIV technique for the monitoring of river surface velocities in high flow conditions. Flow Measurement and Instrumentation. 19(2) 117-127

[19] Abdel-Aziz Y., Karara H. (1971) - Direct Linear Transformation from Comparator Coordinates into Object Space Coordinates in Close-Range Photogrammetry. American Society of Photogrammetry, Symposium on Close-Range Photogrammetry, Urbana, Illinois. 1-18

[20] Fujita I., KomURA S. (1994) - Application of video image analysis for measurements of river-surface flows. Proc. Hydraul. Engng, JSCE. 38 733-738

[21] Rantz S. E. (1982) - Measurement and computation of streamflow. Computation of discharge U.S. Geol. Surv. Water Supply Pap., USGS. 2, 2175373 p

[22] Coles D. (1956) - The law of the wake in the turbulent boundary layer. Journal of Fluid Mechanics. 1 191-226

[23] VAN Risn L. (1986) - Mathematical modeling of suspended sediment in non uniform flows. Journal of Hydraulic Engineering. 112(6) 433-455

[24] Fulford J. M., SAUER V. B. (1986) - Comparison of velocity interpolation methods for computing open-channel discharge. U.S. Geol. Surv. Water Supply Pap. 2290, USGS. 139-144

[25] Olivier A., Blanquart B., Pierrefeu G., Scotti M. (2008) - Incertitude sur les débits calculés à partir de courbes de transformation hauteur/débit. Colloque SHF : Mesures hydrologiques et incertitudes, 1-2 avril 2008, Paris, France.

[26] Hauet A., Creutin J.-D., Belleudy Ph. (2008) - Sensitivity study of large-scale particle image velocimetry measurement of river discharge using numerical simulation. Journal of Hydrology. 349(1-2) 178-190 